J. Nonlinear Var. Anal. 5 (2021), No. 2, pp. 211-226

Available online at http://jnva.biemdas.com

https://doi.org/10.23952/jnva.5.2021.2.03

\title{
A PARAMETERIZED THREE-OPERATOR SPLITTING ALGORITHM AND ITS EXPANSION
}

\author{
CUIJIE ZHANG*, JIAJIA CHEN
}

College of Science, Civil Aviation University of China, Tianjin 300300, China

\begin{abstract}
Based on the parameterized Douglas-Rachford algorithm and the three-operator splitting algorithm, we propose the parameterized three-operator splitting algorithm for finding the least norm solution for the sum of three maximally monotone operators, with one of which is a cocoercive operator. In order to improve the convergence speed of the parameterized three-operator splitting algorithm, the inertial version of the algorithm is studied. In addition, two convergence theorems are established under some conditions. Finally, we illustrate the feasibility and superiority of our algorithm via a numerical example.
\end{abstract}

Keywords. Inertial algorithm; Maximally monotone operator; Resolvent; Superiority; Splitting algorithm.

\section{INTRODUCTION}

Let $H$ be a real Hilbert space with inner product $\langle\cdot, \cdot\rangle$ and induced norm $\|\cdot\|=\sqrt{\langle\cdot, \cdot\rangle}$. Let $T$ be an operator on $H$. We denote the fixed point set of $T$ by Fix $(T)$, and the zero point set of $T$ by $\operatorname{Zer}(T)$. Let $I$ denote the identity operator on $H$. Recall the following definitions. $T: H \rightarrow H$ is said to be $L$-Lipshcitz continuous iff $\|T x-T y\| \leq L\|x-y\|$, where $L>0$ is Lipschitz constant, for all $x, y \in H$. $T: H \rightarrow H$ is said to be nonexpansive iff $L=1$ in the above inequality. $T: H \rightarrow H$ is said to be $\alpha$-averaged iff

$$
T=(1-\alpha) I+\alpha S,
$$

where $S$ is a nonexpansive operator on $H$ and $\alpha$ is a real number in $(0,1) . T: H \rightarrow H$ is said to be firmly nonexpansive iff $2 T-I$ is nonexpansive, or equivalently, for all $x, y \in H$,

$$
\langle T x-T y, x-y\rangle \geq\|T x-T y\|^{2} .
$$

If $T$ is firmly nonexpansive, then $I-T$ is also firmly nonexpansive. If $\operatorname{Fix}(T) \neq \varnothing$, we have that

$$
\langle x-T x, T x-y\rangle \geq 0, \quad \forall x \in H, y \in F i x(T) .
$$

$T: H \rightarrow H$ is said to be monotone iff, for all $x, y \in H,\langle T x-T y, x-y\rangle \geq 0 . T: H \rightarrow H$ is said to be $\beta$-cocoercive (inverse-strongly monotone) iff there exists a positive constant $\beta$ such that

$$
\langle x-y, T x-T y\rangle \geq \beta\|T x-T y\|^{2}, \quad \forall x \in H, y \in H .
$$

\footnotetext{
*Corresponding author.
}

E-mail address: cuijie_zhang@126.com (C. Zhang).

Received December 28, 2020; Accepted February 4, 2021.

(C)2021 Journal of Nonlinear and Variational Analysis 
Recall that a set-valued operator $A: H \rightarrow 2^{H}$ is said to be monotone iff

$$
\langle u-v, x-y\rangle \geq 0, \quad \forall u \in A(x), v \in A(y) .
$$

Further, it is said to be maximally monotone iff, in addition, its graph

$$
\operatorname{Graph}(A):=\{(x, y) \in H \times H: y \in A(x)\}
$$

is not properly contained in the graph of any other monotone operator. It is well-known that a monotone mapping $A$ is maximal if and only if, for $(x, y) \in H \times H,\langle x-v, y-w\rangle \geq 0$ for every $(v, w) \in \operatorname{Graph}(A)$ implies $y \in A(x)$.

Let $A$ be a set-valued maximally monotone operator. Define define the single-valued operator $J_{A}$ by $J_{A}=(I+A)^{-1}$, which is called the resolvent operator of $A$. It is known that $\operatorname{Fix}\left(J_{A}\right)=$ $\operatorname{Zer}(A)$ and the resolvent is firmly nonexpansive. We can also identify the reflected resolvent via $R_{A}=2 J_{A}-I$.

Let $A, B: H \rightarrow 2^{H}$ be two maximally monotone operators and let $C$ be a cocoercive operator on $H$. In this paper, we consider the zero point problem of the sum of three operators, which consists of

$$
\text { finding } x \in H \text { such that } 0 \in A x+B x+C x \text {. }
$$

Problem (1.1), which finds a number of real applications in signal processing, image recovery and machine learning, arises in many fields of fundamental importance in mathematical optimization, such as, saddle point problems, equilibrium problems, variational inequality problems; see, e.g., $[1,2,3,4,5]$. If $C \equiv 0$, then problem (1.1) is reduced to the classical sum problem of two operators, which consists of

$$
\text { finding } x \in H \text { such that } 0 \in A x+B x \text {. }
$$

Splitting methods, which involve with the individual operators only instead of the sum of operators, are popular and efficient to solve the above inclusion problems. The classical DouglasRachford splitting algorithm [6] was introduced to solve problem (1.2) in 1956. It reads as follows

$$
\left\{\begin{array}{l}
y_{k}=J_{B} x_{k}, \\
z_{k}=J_{A}\left(2 y_{k}-x_{k}\right), \\
x_{k+1}=x_{k}+\lambda_{k}\left(z_{k}-y_{k}\right) .
\end{array}\right.
$$

Under some appropriate conditions, it was proved that the sequence $\left\{x_{k}\right\}$ generated by (1.3) converges to a point $x$ in $F i x\left(R_{A}\right)$, and the sequences $\left\{y_{k}\right\}$ and $\left\{z_{k}\right\}$ converge to $J_{B} x$.

Recently, based on the splitting algorithm, many authors investigated various modifications for zero points of maximal monotone operators; see, e.g., [7, 8, 9, 10, 11, 12, 13] and the references therein.

In 2017, Wang and Wang [14] proposed a parameterized Douglas-Rachford splitting algorithm in Euclidean spaces for finding the least norm solution for the sum of two maximally monotone operators. Their algorithm reads as follows

$$
\left\{\begin{array}{l}
y_{k}=J_{B} x_{k}, \\
z_{k}=J_{A}\left(\alpha y_{k}-x_{k}\right), \\
x_{k+1}=x_{k}+\lambda_{k}\left(z_{k}-y_{k}\right),
\end{array}\right.
$$


where $\alpha \in(1,2)$, and $A$ and $B$ are set-valued maximally monotone operators with $0 \in \operatorname{ri}(\operatorname{dom} A-$ $\operatorname{dom} B)$ and $\operatorname{Zer}(A+B) \neq \emptyset$. They showed that there exists $x^{\alpha} \in F i x\left(R_{A}^{\alpha} R_{B}^{\alpha}\right)$, where $R_{A}^{\alpha}=\alpha J_{A}-I$ and $R_{B}^{\alpha}=\alpha J_{B}-I$, such that $\left\{J_{B} x^{\alpha}\right\}=\operatorname{Zer}(A+B+(2-\alpha) I)$ is a singleton, the sequence $\left\{x_{k}\right\}$ generated by (1.4) converges to $x^{\alpha}$, and the sequences $\left\{y_{k}\right\}$ and $\left\{z_{k}\right\}$ converge to $J_{B} x^{\alpha}$.

On the other hand, problem (1.1) was also investigated by many authors and various efficient algorithms were introduced; see, e.g., [15, 16, 17, 18, 19, 20] and the references therein. In 2015, Briceño-Arias [15] proposed a forward-Douglas-Rachford splitting algorithm to solve problem (1.1) with the fact that $B$ is the normal cone to $V$, where $V$ is a closed vector subspace of $H$. In 2017, Davis and Yin [17], based on the Douglas-Rachford algorithm and the forward-backward algorithm, proposed the following three-operator splitting algorithm

$$
\left\{\begin{array}{l}
y_{k}=J_{\gamma B} x_{k}, \\
z_{k}=J_{\gamma A}\left(2 y_{k}-x_{k}-\gamma C y_{k}\right), \\
x_{k+1}=x_{k}+\lambda_{k}\left(z_{k}-y_{k}\right),
\end{array}\right.
$$

where $J_{\gamma A}=(I+\gamma A)^{-1}$ and $J_{\gamma B}=(I+\gamma B)^{-1}$ with $\gamma$ being some positive real number, and $\left\{\lambda_{k}\right\}$ is a sequence of relaxation parameters. Under some appropriate conditions, the sequence $\left\{x_{k}\right\}$ generated by (1.5) converges weakly to a point $x$ in $\operatorname{Fix}\left(T_{D F}\right)$, and the sequences $\left\{y_{k}\right\},\left\{z_{k}\right\}$ converge weakly to $J_{\gamma B} x$ in $J_{\gamma B}\left(F i x\left(T_{D F}\right)\right)$, where

$$
T_{D F}=J_{\gamma A} \circ\left(2 J_{\gamma B}-I-\gamma C \circ J_{\gamma B}\right)+\left(I-J_{\gamma B}\right) \text { and } J_{\gamma B}\left(F i x\left(T_{D F}\right)\right)=\operatorname{Zer}(A+B+C) .
$$

To speed up the convergence of iterative algorithms, the inertial extrapolation technique, which originates from the heavy ball method [21], has been investigated extensively; see, e.g., $[22,23,24,25,26]$ and the references therein. In 2019, Cui, Tang and Yang [16] proposed the following inertial three-operator splitting algorithm

$$
\left\{\begin{array}{l}
w_{k}=x_{k}+\alpha_{k}\left(x_{k}-x_{k-1}\right) \\
y_{k}=J_{\gamma B} w_{k} \\
z_{k}=J_{\gamma A}\left(2 y_{k}-w_{k}-C y_{k}\right) \\
x_{k+1}=w_{k}+\lambda_{k}\left(z_{k}-y_{k}\right) .
\end{array}\right.
$$

They analyzed the convergence of the proposed iterative algorithm and showed the three-operator algorithm with the inertial term is faster than one without the inertial term.

Motivated and inspired by the above results, we propose a parameterized three-operator algorithm and an inertial parameterized three-operator splitting algorithm, which provide a principle of finding the least norm solution for the sum of three maximally monotone operators with one of them is cocoercive. We organize this paper as follows. In Section 2, we recall some lemmas, which will be used in main results. In Section 3, we propose our parameterized three-operator splitting algorithm and analyze the convergence. In Section 4, we introduce our inertial parameterized three-operator splitting algorithm and prove its convergence. In the last section, Section 5, we illustrate the feasibility and superiority of our algorithms via a numerical example.

\section{PRELIMinaries}

In this section, we list some necessary lemmas, which will be used for our main convergence theorems. 
Lemma 2.1. [27] Let $H$ be a Hilbert space. Let $T: H \rightarrow H$ be a nonexpansive operator with Fix $(T) \neq \varnothing$. Let $\left\{x_{k}\right\}$ be a sequence generated by

$$
\left\{\begin{array}{l}
y_{k}=x_{k}+\alpha_{k}\left(x_{k}-x_{k-1}\right), \\
x_{k+1}=\left(1-\lambda_{k}\right) y_{k}+\lambda_{k} T y_{k},
\end{array}\right.
$$

where $\left\{\lambda_{k}\right\} \subset(0,1)$ and $\left\{\alpha_{k}\right\} \subset[0,1)$ satisfy the following conditions:

(i) $\left\{\alpha_{k}\right\}_{k \geq 1}$ is nondecreasing with $\alpha_{1}=0$ and $0 \leq \alpha_{k} \leq \alpha<1$ for every $k \geq 1$;

(ii) Let $\lambda, \rho, \sigma>0$ such that

$$
\rho>\frac{\delta^{2}(1+\delta)+\delta \sigma}{1-\delta^{2}} \text { and } 0<\lambda \leq \lambda_{k} \leq \frac{\rho-\delta[\delta(1+\delta)+\delta \rho+\sigma]}{\rho[1+\delta(1+\delta)+\delta \rho+\sigma]}
$$

Then the following assertions hold:

(a) For any $x^{*} \in \operatorname{Fix}(T), \lim _{k \rightarrow+\infty}\left\|x_{k}-x^{*}\right\|$ exists;

(b) $\sum_{k=0}^{\infty}\left\|x_{k+1}-x_{k}\right\|^{2}<+\infty$;

(c) $\left\{x_{k}\right\}$ converges weakly to a point in Fix $(T)$.

Lemma 2.2. [28] Let $H$ be a Hilbert space. Let $\left\{x_{n}\right\}_{n \in N}$ be a sequence in H. Then $\left\{x_{n}\right\}_{n \in N}$ converges weakly in $H$ if and only if it is bounded and possesses at most one weak sequential cluster point in $H$.

Lemma 2.3. [28] Let $H$ be a Hilbert space. Let $A: H \rightarrow 2^{H}$ be a maximally monotone operator. Let $\left(x_{b}, u_{b}\right)_{b \in N}$ be a bounded net in Graph $(A)$, where Graph $(A)$ denotes the graph of $A$, and let $(x, u) \in H \times H$. Then, the following assertions hold:

(i) If $x_{b} \rightarrow x$ and $u_{b} \rightarrow u$, then $(x, u) \in \operatorname{Graph}(A)$;

(ii) If $x_{b} \rightarrow x$ and $u_{b} \rightarrow u$, then $(x, u) \in \operatorname{Graph}(A)$.

Lemma 2.4. [28] Let $m$ be an integer such that $m \geq 2$, and set $M=1, \cdots, m$. Let $\left(A_{i}\right)_{i \in M}$ be maximally monotone operators from $H$ to $2^{H}$. For every $i \in M$, let $\left(x_{i, n}, u_{i, n}\right)_{n \in N}$ be a sequence in $\operatorname{Graph}\left(A_{i}\right)$ and let $\left(x_{i}, u_{i}\right) \in H \times H$. Suppose that $\sum_{i \in M} u_{i, n} \rightarrow 0$, and

$$
\left\{\begin{array}{l}
x_{i, n} \rightarrow x_{i} \\
u_{i, n} \rightarrow u_{i} \\
m x_{i, n}-\sum_{j \in M} x_{j, n} \rightarrow 0 .
\end{array}\right.
$$

Then there exists $x \in \operatorname{Zer} \sum_{i \in M} A_{i}$ such that the following hold:

(a) $x=x_{1}=\cdots=x_{m}$;

(b) $\sum_{i \in M} u_{i}=0$;

(c) $(\forall i \in M)\left(x_{i}, u_{i}\right) \in \operatorname{Graph}\left(A_{i}\right)$;

(d) $\sum_{i \in M}\left\langle x_{i, n}, u_{i, n}\right\rangle \rightarrow\left\langle x, \sum_{i \in M} u_{i}\right\rangle=0$.

Lemma 2.5. [28] Let $H$ be a Hilbert space. Let $A$ and $B$ be maximally monotone operators from $H$ to $2^{H}$ such that one of the following holds:

(i) $\operatorname{dom} A \cap \operatorname{ri}(\operatorname{dom} B) \neq \varnothing$;

(ii) $0 \in \operatorname{ri}(\operatorname{dom} A-\operatorname{dom} B)$.

Then $A+B$ is maximally monotone operator. 
Lemma 2.6. [28] Let $H$ be a Hilbert space. Let $A$ be a maximally monotone operator from $H$ to $2^{H}$, and let $x \in H$ and $\gamma \in(0,1)$. Then the inclusion $0 \in A x_{\gamma}+\gamma\left(x_{\gamma}-x\right)$ defines a unique curve $\left\{x_{\gamma}\right\}$. Moreover, exactly one of the following holds:

(i) ZerA $\neq \varnothing$ and $x_{\gamma} \rightarrow P_{Z e r A} x$ as $\gamma \downarrow 0$;

(ii) $\operatorname{Zer} A=\varnothing$ and $\left\|x_{\gamma}\right\| \rightarrow+\infty$ as $\gamma \downarrow 0$.

Lemma 2.7. [28] Let $H$ be a Hilbert space. Let $A: H \rightarrow 2^{H}$ be a monotone operator. Then $A$ is maximally monotone if and only if $\operatorname{Ran}(I+A)=H$, where $\operatorname{Ran}(I+A)$ denotes the range of $I+A$.

Lemma 2.8. [29] Let $H$ be a Hilbert space. Let $A: H \rightarrow 2^{H}$ be a maximally monotone operator and let $f: H \rightarrow H$ be a Lipschitz continuous operator. Then the operator $B=A+f$ is maximally monotone.

Lemma 2.9. [28] Let $H$ be a Hilbert space and let $D$ be a nonempty subset of $H$. Let $T: D \rightarrow H$ be a nonexpensive operator, and let $\alpha \in(0,1)$. Then the following assertions are equivalent:

(a) $T$ is $\alpha$-averaged;

(b) $\left(1-\frac{1}{\alpha}\right) I+\left(\frac{1}{\alpha}\right) T$ is nonexpansive;

(c) $(\forall x \in D)(\forall y \in D)\|T x-T y\|^{2} \leq\|x-y\|^{2}-\frac{1-\alpha}{\alpha}\|(I-T) x-(I-T) y\|^{2}$;

(d) $(\forall x \in D)(\forall y \in D)\|T x-T y\|^{2}+(1-2 \alpha)\|x-y\|^{2} \leq 2(1-\alpha)\langle x-y, T x-T y\rangle$.

\section{Algorithm AND CONVERGENCE ANALYSIS}

In this section, we introduce the parameterized three-operator splitting algorithm and investigate its convergence.

3.1. The parameterized three-operator splitting algorithm. Let us start with three hypotheses.

Condition (i) $A$ is a maximally monotone operator.

Condition (ii) $B$ is a maximally monotone operator.

Condition (iii) $C$ is a $\beta$-cocoercive operator.

Algorithm 3.1. Initialization: Set $z_{0} \in H, 0<\gamma<\min \left\{\frac{1}{2-\alpha}, \beta\right\}$ and sequence $\left\{\lambda_{k}\right\}_{k \geq 0} \in$ $\left(0,2-2 \max \left\{\frac{\gamma}{2 \beta}, \frac{\gamma(2-\alpha)}{2}\right\}\right)$.

Iterative steps: For $k=0,1, \cdots$, iterate:

Step 1. Compute

$$
x_{k}=J_{\gamma B}\left(z_{k}\right)
$$

Step 2. Compute

$$
y_{k}=J_{\gamma A}\left\{[2-\gamma(2-\alpha)] x_{k}-z_{k}-\gamma C x_{k}\right\} .
$$

Step 3. Compute

$$
z_{k+1}=z_{k}+\lambda_{k}\left(y_{k}-x_{k}\right) .
$$

Now we introduce the following operator

$$
T:=J_{\gamma A} \circ\left\{[2-\gamma(2-\alpha)] J_{\gamma B}-I-\gamma C \circ J_{\gamma B}\right\}+I-J_{\gamma B} .
$$


We rewrite the above algorithm as the Krasnosel'skiı̆-Mann (KM) iteration

$$
z_{k+1}=\left(1-\lambda_{k}\right) z_{k}+\lambda_{k} T z_{k} .
$$

If $T$ has a fixed-point, our KM iteration converges weakly to a fixed-point of $T$ at the fixed-point rate $\left\|T z_{k}-z_{k}\right\|^{2}=o\left((k+1)^{-1}\right)$.

Remark 3.1. Based on Algorithm 3.1, we have the following facts. If $C=0$ and $\gamma=1$, then $T$ becomes the $\alpha$-Douglas-Rachford operator $T_{D R}^{\alpha}:=J_{\gamma A} \circ\left(\alpha J_{\gamma B}-I\right)+I-J_{\gamma B}$; if $\alpha \rightarrow 2$, then $T$ becomes the three-operator $T_{D F}:=J_{\gamma A} \circ\left(2 J_{\gamma B}-I-\gamma C \circ J_{\gamma B}\right)+I-J_{\gamma B}$; if $\alpha \rightarrow 2$ and $B=0$, then $T$ becomes the forward-backward operator $T_{F B}:=J_{\gamma A} \circ(I-\gamma C)$; if $A \leftarrow 0, C \leftarrow A$, and $\alpha \rightarrow 2$, then $T$ becomes the backward-forward operator $T_{B F}:=(I-\gamma A) \circ J_{\gamma B}$.

Lemma 3.1. Let $z \in H$ and define points: $x_{k}:=J_{\gamma B}\left(z_{k}\right), m_{k}:=[2-\gamma(2-\alpha)] x_{k}-z_{k}, n_{k}:=$ $m_{k}-\gamma C x_{k}, y_{k}:=J_{\gamma A}\left(n_{k}\right), u_{k}:=\gamma^{-1}\left(z_{k}-x_{k}\right) \in B x_{k}, v_{k}:=\gamma^{-1}\left(n_{k}-y_{k}\right) \in A y_{k}$. Then the following identities hold:

$$
T z_{k}-z_{k}=y_{k}-x_{k}=-\gamma\left[u_{k}+v_{k}+C x_{k}+(2-\alpha) x_{k}\right] \text { and } T z_{k}=y_{k}+\gamma u_{k} .
$$

Proof. Observe that $T z_{k}=z_{k}+y_{k}-x_{k}$. In addition, $T z_{k}=y_{k}+z_{k}-x_{k}=y_{k}+\gamma u_{k}$. It follows that $y_{k}-x_{k}=[2-\gamma(2-\alpha)] x_{k}-z_{k}-\gamma C x_{k}-\gamma v_{k}-x_{k}=-\gamma\left[v_{k}+u_{k}+C x_{k}+(2-\alpha) x_{k}\right]$. This completes the proof.

Lemma 3.2. (Fixed-point encoding) Let $A, B: H \rightarrow 2^{H}$ be maximally monotone operators with $0 \in \operatorname{ri}(\operatorname{dom} A-\operatorname{dom} B)$ and let $C$ be a cocoercive operator. The

(1) $\operatorname{Zer}[A+B+C+(2-\alpha) I] \neq \varnothing$;

(2) $\operatorname{Zer}[A+B+C+(2-\alpha) I]=J_{\gamma B}[$ Fix $(T)]$ and Fix $(T) \neq \varnothing$;

(3) $\operatorname{Zer}[A+B+C+(2-\alpha) I]$ is a singleton.

Proof. (1) Since $A$ and $B$ are maximally monotone, and $0 \in \operatorname{ri}(\operatorname{dom} A-\operatorname{dom} B)$, we conclude from Lemma 2.5 that $A+B$ is maximally monotone. Since $C$ is $\beta$-cocoercive, we have that $C$ is Lipschitz continuous. From Lemma 2.8, we have that $A+B+C$ is maximally monotone. It follows from Lemma 2.7 that $\operatorname{Ran}\left(I+\frac{1}{2-\alpha}(A+B+C)\right)=H$, which in turn implies that $0 \in \operatorname{Ran}\left(I+\frac{1}{2-\alpha}(A+B+C)\right)$. Hence, there exists $x \in H$ with $0 \in x+\frac{1}{2-\alpha}(A+B+C) x$. It follows that $0 \in A x+B x+C x+(2-\alpha) x$, and $\operatorname{Zer}[A+B+C+(2-\alpha) I] \neq \varnothing$.

(2) From (1), we have $0 \in \gamma A x+\gamma B x+\gamma C x+\gamma(2-\alpha) x$. Therefore, there exists $y \in H$ such that $x-y \in \gamma A x+\gamma C x+\gamma(2-\alpha) x$ and $y-x \in \gamma B x$, which is equivalent to $[1-\gamma(2-\alpha)] x-$ $y-\gamma C x \in \gamma A x$ and $x=J_{\gamma B} y$. It follows that $[2-\gamma(2-\alpha)] x-y-\gamma C x \in(I+\gamma A) x$, and then $y=J_{\gamma A}\left\{\left([2-\gamma(2-\alpha)] J_{\gamma B}-I-\gamma C J_{\gamma B}\right) y\right\}-J_{\gamma B} y+y$, that is $y=T y$. Note that $x=J_{\gamma B} y, y \in$ Fix $(T), x \in \operatorname{Zer}[A+B+C+(2-\alpha) I]$ and that the process can be reversed. So, we have $J_{\gamma B}(F i x(T))=\operatorname{Zer}[A+B+C+(2-\alpha) I]$.

(3) If, $\alpha \in(0,2), 2-\alpha>0$, then $A+B+C+(2-\alpha) I$ is strictly monotone. Hence, $\operatorname{Zer}[A+$ $B+C+(2-\alpha) I]$ is singleton.

\subsection{Convergence analysis.}

Lemma 3.3. [17] Let $S:=U+T_{1} \circ V$, where $U, T_{1}: H \rightarrow H$ are both firmly nonexpansive and $V: H \rightarrow H$. Let $W=I-(2 U+V)$. Then, we have for all $z, w \in H$ :

$$
\|S z-S w\|^{2} \leq\|z-w\|^{2}-\|(I-S) z-(I-S) w\|^{2}-2\left\langle T_{1} \circ V z-T_{1} \circ V w, W z-W w\right\rangle .
$$


The above Lemma is crucial for proving the convergence of Algorithm 3.1. The following Lemma show that $T$ is averaged.

Lemma 3.4. (Averageness of $T$ ) Suppose that $T_{1}, T_{2}: H \rightarrow H$ are firmly nonexpansive and $C$ is $\beta$-cocoercive with $\beta>0$. Then

$$
T:=I-T_{2}+T_{1} \circ\left\{[2-\gamma(2-\alpha)] T_{2}-I-\gamma C \circ T_{2}\right\}
$$

is averaged.

Proof. In view of Lemma 3.3, we let $U=I-T_{2}, V=[2-\gamma(2-\alpha)] T_{2}-I-\gamma C \circ T_{2}$ and $W=$ $\gamma(2-\alpha) T_{2}+\gamma C \circ T_{2}$. Note that $U$ is firmly nonexpansive and $W=I-(2 U+V)$. Letting $S=T=I-T_{2}+T_{1} \circ V$, we evaluate the inner product in (3.2) as follows:

$$
\begin{aligned}
- & 2\left\langle T_{1} \circ V z-T_{1} \circ V w, W z-W w\right\rangle \\
= & 2\langle(I-T) z-(I-T) w, W z-W w\rangle-2\left\langle T_{2} z-T_{2} w, W z-W w\right\rangle \\
= & 2\left\langle(I-T) z-(I-T) w, \gamma(2-\alpha) T_{2} z-\gamma(2-\alpha) T_{2} w\right\rangle \\
& -2\left\langle T_{2} z-T_{2} w, \gamma(2-\alpha) T_{2} z-\gamma(2-\alpha) T_{2} w\right\rangle \\
& +2\left\langle(I-T) z-(I-T) w, \gamma C \circ T_{2} z-\gamma C \circ T_{2} w\right\rangle-2\left\langle T_{2} z-T_{2} w, \gamma C \circ T_{2} z-\gamma C \circ T_{2} w\right\rangle \\
\leq & -2 \gamma \beta\left\|C \circ T_{2} z-C \circ T_{2} w\right\|^{2}+\varepsilon\|(I-T) z-(I-T) w\|^{2}+\frac{\gamma^{2}}{\varepsilon}\left\|C \circ T_{2} z-C \circ T_{2} w\right\|^{2} \\
& -2 \gamma(2-\alpha)\left\|T_{2} z-T_{2} w\right\|^{2}+\varepsilon\|(I-T) z-(I-T) w\|^{2}+\frac{\gamma^{2}(2-\alpha)^{2}}{\varepsilon}\left\|T_{2} z-T_{2} w\right\|^{2} \\
= & 2 \varepsilon\|(I-T) z-(I-T) w\|^{2}-\gamma\left(2 \beta-\frac{\gamma}{\varepsilon}\right)\left\|C \circ T_{2} z-C \circ T_{2} w\right\|^{2} \\
& -\gamma(2-\alpha)\left[2-\frac{\gamma(2-\alpha)}{\varepsilon}\right]\left\|T_{2} z-T_{2} w\right\|^{2},
\end{aligned}
$$

where the inequality follows from Young's inequality with any $\varepsilon>0$, and $C$ is $\beta$-cocoercive. Let

$$
0<\max \left\{\frac{\gamma}{2 \beta}, \frac{\gamma(2-\alpha)}{2}\right\} \leq \varepsilon<\frac{1}{2} .
$$

So, $\gamma\left(2 \beta-\frac{\gamma}{\varepsilon}\right) \geq 0$ and $2-\frac{\gamma(2-\alpha)}{\varepsilon} \geq 0$. Using Lemma 3.3 and $S=T$, we obtain

$$
\begin{aligned}
\|T z-T w\|^{2} \leq & \|z-w\|^{2}-(1-2 \varepsilon)\|(I-T) z-(I-T) w\|^{2} \\
& -\gamma\left(2 \beta-\frac{\gamma}{\varepsilon}\right)\left\|C \circ T_{2} z-C \circ T_{2} w\right\|^{2}-\gamma(2-\alpha)\left[2-\frac{\gamma(2-\alpha)}{\varepsilon}\right]\left\|T_{2} z-T_{2} w\right\|^{2} .
\end{aligned}
$$

Then $T$ is $\theta$-averaged, where $\theta=\frac{1}{2-2 \varepsilon}$.

Remark 3.2. (1) For any $\bar{\varepsilon} \in\left(0, \frac{1}{2}\right)$ and $0<\gamma<\min \left\{2 \beta \bar{\varepsilon}, \frac{2 \bar{\varepsilon}}{2-\alpha}\right\}$, let $\bar{\theta}=\frac{1}{2-2 \bar{\varepsilon}}<1$. Then, for all $z, w \in H$,

$$
\begin{aligned}
\|T z-T w\|^{2} & \leq\|z-w\|^{2}-\frac{1-\bar{\theta}}{\bar{\theta}}\|(I-T) z-(I-T) w\|^{2} \\
& -\gamma\left(2 \beta-\frac{\gamma}{\bar{\varepsilon}}\right)\left\|C \circ T_{2} z-C \circ T_{2} w\right\|^{2}-\gamma(2-\alpha)\left[2-\frac{\gamma(2-\alpha)}{\bar{\varepsilon}}\right]\left\|T_{2} z-T_{2} w\right\|^{2} .
\end{aligned}
$$

(2) If $\alpha$ is very close to 2 , we can choose $\varepsilon=\frac{\gamma}{2 \beta}$ in (3.4) to obtain that $T$ is $\frac{\beta}{2 \beta-\gamma}$-averaged. 
Corollary 3.1. Set a stepsize $\gamma \in\left(0, \min \left\{2 \beta \bar{\varepsilon}, \frac{2 \bar{\varepsilon}}{2-\alpha}\right\}\right)$, where $\bar{\varepsilon} \in\left(0, \frac{1}{2}\right)$. Set $\left\{\lambda_{k}\right\}_{k \geq 0} \subseteq\left(0, \frac{1}{\bar{\theta}}\right)$ as a sequence of relaxation parameters, where $\bar{\theta}=\frac{1}{2-2 \bar{\varepsilon}}$ such that, for $\tau_{k}:=\left(1-\lambda_{k} \bar{\theta}\right) \frac{\lambda_{k}}{\bar{\theta}}$, $\Sigma_{k=0}^{\infty} \tau_{k}=\infty$. Pick an initial $z_{0} \in H$, and let $\left\{z_{k}\right\}_{k \geq 0}$ be generated by Algorithm 3.1. Then,

(1) for $z^{*} \in \operatorname{Fix}(T),\left\{\left\|z_{k}-z^{*}\right\|\right\}_{k \geq 0}$ is monotonically nonincreasing;

(2) the fixed-point residual sequence $\left\{\left\|T z_{k}-z_{k}\right\|\right\}_{k \geq 0}$ is monotonically nonincreasing and converges to 0 ;

(3) the sequence $\left\{z_{k}\right\}_{k \geq 0}$ converges weakly to a fixed point of $T$, which we denote as $z^{*}$, at the same time, $J_{\gamma B}\left(z^{*}\right)$ in $\operatorname{Zer}[A+B+C+(2-\alpha) I]$;

(4) suppose that $\tau:=\inf _{k \geq 0} \tau_{k}>0$. Then, for all $k \geq 0$, the following convergence rates hold:

$$
\left\|T z_{k}-z_{k}\right\|^{2} \leq \frac{\left\|z_{0}-z^{*}\right\|^{2}}{\underline{\tau}(k+1)} \text { and }\left\|T z_{k}-z_{k}\right\|^{2}=o\left(\frac{1}{k+1}\right),
$$

for any point $z^{*} \in \operatorname{Fix}(T)$.

Theorem 3.1. Set a stepsize $\gamma \in\left(0, \min \left\{2 \beta \bar{\varepsilon}, \frac{2 \bar{\varepsilon}}{2-\alpha}\right\}\right)$, where $\bar{\varepsilon} \in\left(0, \frac{1}{2}\right)$. Set $\left\{\lambda_{k}\right\}_{k \geq 0} \subseteq\left(0, \frac{1}{\bar{\theta}}\right)$ as a sequence of relaxation parameters where $\bar{\theta}=\frac{1}{2-2 \bar{\varepsilon}}$, such that, for $\tau_{k}:=\left(1-\lambda_{k} \bar{\theta}\right) \frac{\lambda_{k}}{\bar{\theta}}, \Sigma_{k=0}^{\infty} \tau_{k}=\infty$. Pick an initial $z_{0} \in H$, and let $\left\{z_{k}\right\}_{k \geq 0}$ be generated by Algorithm 3.1. Let $\inf _{k \geq 0} \lambda_{k}=\underline{\lambda}>0$ and let $z^{*}$ be the weak limit of $z_{k}$ (which exists by Corollary 3.1). Then,

(1) $\left\{C\left(x_{k}\right)\right\}_{k \geq 0}$ converges strongly to $C J_{\gamma B}\left(z^{*}\right)$ for $J_{\gamma B}\left(z^{*}\right) \in \operatorname{Zer}[A+B+C+(2-\alpha) I]$;

(2) the sequence $\left\{x_{k}\right\}_{k \geq 0}$ converges weakly to $J_{\gamma B}\left(z^{*}\right) \in \operatorname{Zer}[A+B+C+(2-\alpha) I]$;

(3) the sequence $\left\{y_{k}\right\}_{k \geq 0}$ converges weakly to $J_{\gamma B}\left(z^{*}\right) \in \operatorname{Zer}[A+B+C+(2-\alpha) I]$.

Proof. (1) Fix $k \geq 0$. Observe that

$$
\left\|z_{k+1}-z^{*}\right\|^{2}=\left(1-\lambda_{k}\right)\left\|z_{k}-z^{*}\right\|^{2}+\lambda_{k}\left\|T z_{k}-z^{*}\right\|^{2}-\lambda_{k}\left(1-\lambda_{k}\right)\left\|T z_{k}-z_{k}\right\|^{2} .
$$

In addition, from (3.5), we have

$$
\begin{gathered}
\left\|T z_{k}-z^{*}\right\|^{2} \leq\left\|z_{k}-z^{*}\right\|^{2}-\frac{1-\bar{\theta}}{\bar{\theta}}\left\|T z_{k}-z_{k}\right\|^{2}-\gamma\left(2 \beta-\frac{\gamma}{\bar{\varepsilon}}\right)\left\|C \circ T_{2} z_{k}-C \circ T_{2} z^{*}\right\|^{2} \\
-\gamma(2-\alpha)\left[2-\frac{\gamma(2-\alpha)}{\bar{\varepsilon}}\right]\left\|T_{2} z_{k}-T_{2} z^{*}\right\|^{2}
\end{gathered}
$$

In view of (3.6), (3.7) and

we arrive at

$$
\tau_{k}=\lambda_{k}\left(1-\lambda_{k}\right)+\frac{\lambda_{k}(1-\bar{\theta})}{\bar{\theta}}
$$

$$
\begin{aligned}
& \left\|z_{k+1}-z^{*}\right\|^{2}+\tau_{k}\left\|T z_{k}-z_{k}\right\|^{2}+\gamma \lambda_{k}\left(2 \beta-\frac{\gamma}{\bar{\varepsilon}}\right)\left\|C x_{k}-C J_{\gamma B}\left(z^{*}\right)\right\|^{2} \\
& \quad+\gamma \lambda_{k}(2-\alpha)\left[2-\frac{\gamma(2-\alpha)}{\bar{\varepsilon}}\right]\left\|T_{2} z_{k}-T_{2} z^{*}\right\|^{2} \\
& \leq\left\|z_{k}-z^{*}\right\|^{2} .
\end{aligned}
$$

It follows that

$$
\gamma \lambda_{k}\left(2 \beta-\frac{\gamma}{\bar{\varepsilon}}\right)\left\|C x_{k}-C J_{\gamma B}\left(z^{*}\right)\right\|^{2} \leq\left\|z_{k}-z^{*}\right\|^{2}-\left\|z_{k+1}-z^{*}\right\|^{2}
$$


and then

$$
\sum_{k=0}^{\infty}\left\|C x_{k}-C J_{\gamma B}\left(z^{*}\right)\right\|^{2} \leq \frac{\left\|z_{0}-z^{*}\right\|^{2}}{\gamma \underline{\lambda}\left(2 \beta-\frac{\gamma}{\bar{\varepsilon}}\right)} .
$$

This finds that $\left\|C x_{k}-C J_{\gamma B}\left(z^{*}\right)\right\|^{2} \rightarrow 0$ as $k \rightarrow \infty$.

(2) Set $x_{k}=J_{\gamma B}\left(z_{k}\right), y_{k}=J_{\gamma A}\left\{[2-\gamma(2-\alpha)] x_{k}-z_{k}-\gamma C x_{k}\right\}, u_{k}=\frac{1}{\gamma}\left(z_{k}-x_{k}\right) \in B x_{k}$, and $v_{k}=\frac{1}{\gamma}\left\{[2-\gamma(2-\alpha)] x_{k}-z_{k}-\gamma C x_{k}-y_{k}\right\} \in A y_{k}$. From Corollary 3.1, we have

$$
\left\|x_{k}-J_{\gamma B}\left(z^{*}\right)\right\|=\left\|J_{\gamma B}\left(z_{k}\right)-J_{\gamma B}\left(z^{*}\right)\right\| \leq\left\|z_{k}-z^{*}\right\| \leq\left\|z_{0}-z^{*}\right\|
$$

for all $k \geq 0$. Then, $\left\{x_{k}\right\}_{k \geq 0}$ is bounded. Therefore, there exists a subsequence $\left\{x_{k_{j}}\right\}$ and a weak sequential cluster point $\underline{x}$ such that $x_{k_{j}} \rightarrow \underline{x}$. Let $x^{*} \in \operatorname{Zer}[A+B+C+(2-\alpha) I]$. From Lemma 3.2 (3), we obtain $x^{*}=J_{\gamma B}\left(z^{*}\right)$. Since $C$ is maximally monotone, $C x_{k} \rightarrow C x^{*}$ and $x_{k_{j}} \rightarrow \underline{x}$, we conclude from the weak-to-strong sequential closeness of $C$ that $C \underline{x}=C x^{*}$. Thus $C x_{k_{j}} \rightarrow C \underline{x}$. In view of $y_{k}-x_{k}=T z_{k}-z_{k} \rightarrow 0$ as $k \rightarrow \infty$, Corollary 3.1 (2) and Lemma 3.1 guarantee that

$$
\begin{aligned}
& x_{k_{j}} \rightarrow \underline{x}, \quad y_{k_{j}} \rightarrow \underline{x}, \quad u_{k} \rightarrow \frac{1}{\gamma}\left(z^{*}-\underline{x}\right), \\
& v_{k_{j}} \rightarrow \frac{1}{\gamma}\left\{[1-\gamma(2-\alpha)] \underline{x}-z^{*}-\gamma C \underline{x}\right\} \text { as } j \rightarrow \infty .
\end{aligned}
$$

Since $\left(y_{k_{j}}, v_{k_{j}}\right) \in \operatorname{Graph}(A),\left(x_{k_{j}}, u_{k_{j}}\right) \in \operatorname{Graph}(B),\left(x_{k_{j}}, C x_{k_{j}}\right) \in \operatorname{Graph}(C)$ and $\left(x_{k_{j}},(2-\alpha) x_{k_{j}}\right) \in$ $\operatorname{Graph}[(2-\alpha) I]$, with the aid of Lemma 2.4, we obtain that $\underline{x} \in \operatorname{Zer}[A+B+C+(2-\alpha) I]$. It follows that $\underline{x}=J_{\gamma B}\left(z^{*}\right)$, and $\underline{x}$ is the unique weak sequential point of $\left\{x_{k}\right\}_{k \geq 0}$. Therefore $\left\{x_{k}\right\}_{k \geq 0}$ converges weakly to $J_{\gamma B}\left(z^{*}\right)$ by Lemma 2.2.

(3) From $y_{k}-x_{k}=T z_{k}-z_{k} \rightarrow 0$ as $k \rightarrow \infty$ and $x_{k} \rightarrow J_{\gamma B}\left(z^{*}\right)$, we conclude $y_{k} \rightarrow J_{\gamma B}\left(z^{*}\right)$ immediately.

\subsection{The regularization of the parameterized three-operator algorithm.}

Algorithm 3.2. Initialization: Set $z_{0} \in H, 0<\gamma<\min \left\{\frac{1}{2-\alpha}, \beta\right\}$, and sequence $\left\{\lambda_{k}\right\}_{k \geq 0} \in$ $\left(0,2-2 \max \left\{\frac{\gamma}{2 \beta}, \frac{\gamma(2-\alpha)}{2}\right\}\right)$.

Iterative steps: For $k=0,1, \cdots$, iterate:

Step 1. Compute

$$
x_{k}=J_{\gamma B}\left(z_{k}\right)
$$

Step 2. Compute

$$
y_{k}=J_{\gamma A}\left\{\left[2-\gamma\left(2-\alpha_{n}\right)\right] x_{k}-z_{k}-\gamma C x_{k}\right\} .
$$

Step 3. Compute

$$
z_{k+1}=z_{k}+\lambda_{k}\left(y_{k}-x_{k}\right)
$$

Theorem 3.2. Let $A, B$ be maximally monotone operators from $H$ to $2^{H}$ such that Zer $(A+B+$ $C) \neq \varnothing$ and $0 \in \operatorname{ri}(\operatorname{dom} A-\operatorname{dom} B)$. Let $C$ be a cocoercive operator. Moreover, let $\left\{\alpha_{n}\right\}_{n \in N}$ be an increasing sequence in $(1,2)$ such that $\lim _{n \rightarrow+\infty} \alpha_{n}=2$. Let $\left\{x_{k}\right\}_{k \in N},\left\{y_{k}\right\}_{k \in N},\left\{z_{k}\right\}_{k \in N}$ be seequences generated by Algorithm 3.2. Then $\left\{z_{k}\right\}_{k \in N}$ converges to $z_{n}^{*}$ such that $J_{\gamma B} z_{n}^{*}=$ $\operatorname{Zer}\left[A+B+C+\left(2-\alpha_{n}\right) I\right]$ and

(1) $\lim _{n \rightarrow+\infty} J_{\gamma B} z_{n}^{*}=P_{\mathrm{Zer}(A+B+C)}(0)$;

(2) suppose $\left\{z_{n}^{*}\right\}_{n \in N}$ is a convergent sequence, say, $\lim _{n \rightarrow+\infty} z_{n}^{*}=z^{*}$. Then $J_{\gamma B} z^{*}$ is a solution to $0 \in A x+B x+C x$, and $\left\|J_{\gamma B} z^{*}\right\| \leq\|y\|$ for any $y \in \operatorname{Zer}(A+B+C)$. 
Proof. (1) From Theorem 3.1, we have $J_{\gamma B} z_{n}^{*}=\operatorname{Zer}\left[A+B+C+\left(2-\alpha_{n}\right) I\right]$. It follows that

$$
0 \in(A+B+C) J_{\gamma B} z_{n}^{*}+\left(2-\alpha_{n}\right) J_{\gamma B} z_{n}^{*}
$$

which is equivalent to

$$
0 \in(A+B+C) J_{\gamma B} z_{n}^{*}+\left(2-\alpha_{n}\right)\left(J_{\gamma B} z_{n}^{*}-0\right) .
$$

As $\operatorname{Zer}(A+B+C) \neq \varnothing$, we conclude from Lemma 2.6 that

$$
J_{\gamma B} z_{n}^{*} \rightarrow P_{\mathrm{Zer}(A+B+C)}(0) \text { as } \quad\left(2-\alpha_{n}\right) \downarrow 0 .
$$

Since $\lim _{n \rightarrow+\infty} \alpha_{n}=2$, we have

$$
\lim _{n \rightarrow+\infty} J_{\gamma B} z_{n}^{*}=P_{\operatorname{Zer}(A+B+C)}(0) .
$$

(2) If $\lim _{n \rightarrow+\infty} z_{n}^{*}=z^{*}$, then

$$
\lim _{n \rightarrow+\infty} J_{\gamma B} z_{n}^{*}=J_{\gamma B} z^{*}
$$

So, $J_{\gamma B} z^{*}=P_{\operatorname{Zer}(A+B+C)}(0)$.

\section{THE INERTIAL PARAMETERIZED THREE-OPERATOR ALGORITHM}

4.1. The inertial parameterized three-operator algorithm and its convergence analysis. In this subsection, we propose an inertial parameterized three-operator splitting algorithm to solve the zero point problem of the sum of three operators and prove its convergence.

Algorithm 4.1. Initialization: Set $z_{0} \in H, 0<\gamma<\min \left\{\frac{1}{2-\alpha}, \beta\right\},\left\{\lambda_{k}\right\}_{k \geq 0} \in\left(0,2-2 \max \left\{\frac{\gamma}{2 \beta}\right.\right.$, $\left.\left.\frac{\gamma(2-\alpha)}{2}\right\}\right)$, and choose $\left\{\delta_{k}\right\} \in[0,1)$.

Iterative steps: For $k=0,1, \cdots$, iterate:

Step 1. Compute

$$
w_{k}=z_{k}+\delta_{k}\left(z_{k}-z_{k-1}\right) \text {. }
$$

Step 2. Compute

$$
x_{k}=J_{\gamma B}\left(w_{k}\right)
$$

Step 3. Compute

$$
y_{k}=J_{\gamma A}\left\{[2-\gamma(2-\alpha)] x_{k}-w_{k}-\gamma C x_{k}\right\} .
$$

Step 4. Compute

$$
z_{k+1}=w_{k}+\lambda_{k}\left(y_{k}-x_{k}\right) .
$$

If $\delta_{k} \equiv 0$, then the inertial parameterized three-operator splitting algorithm turns to Algorithm 3.1. Now, we are ready to prove the convergence of Algorithm 4.1.

Theorem 4.1. Let $A, B: H \rightarrow 2^{H}$ be two maximally monotone operators and let $C: H \rightarrow H$ be a $\beta$-cocoercive operator with $\beta>0$. Set $\left\{\lambda_{k}\right\}_{k \geq 0} \subseteq\left(0, \frac{1}{\bar{\theta}}\right)$ as a sequence of relaxation parameters such that, for $\tau_{k}:=\left(1-\lambda_{k} \bar{\theta}\right) \frac{\lambda_{k}}{\bar{\theta}}, \Sigma_{k=0}^{\infty} \tau_{k}=\infty$. Let $T:=I-J_{\gamma B}+J_{\gamma A}\left\{[2-\gamma(2-\alpha)] J_{\gamma B}-I-\right.$ $\left.\gamma C \circ J_{\gamma B}\right\}$. Let the iterative sequences $\left\{z_{k}\right\},\left\{x_{k}\right\}$, and $\left\{y_{k}\right\}$ be generated by Algorithm 4.1, where the parameters $\gamma,\left\{\sigma_{k}\right\}$, and $\left\{\lambda_{k}\right\}$ satisfy the following conditions:

(1) $0<\gamma<\min \left\{\frac{2 \bar{\varepsilon}}{2-\alpha}, 2 \beta \bar{\varepsilon}\right\}$, where $\bar{\varepsilon} \in\left(0, \frac{1}{2}\right)$;

(2) $\left\{\delta_{k}\right\}$ is nondecreasing with $\delta_{1}=0$ and $0 \leq \delta_{k} \leq \delta<1$; 
(3) Let $\lambda, \rho$ and $\sigma$ be three positive real numbers such that

$$
\rho>\frac{\delta^{2}(1+\delta)+\delta \sigma}{1-\delta^{2}} \text { and } 0<\lambda \leq \lambda_{k} \leq \frac{\rho-\delta[\delta(1+\delta)+\delta \rho+\sigma]}{\theta \rho[1+\delta(1+\delta)+\delta \rho+\sigma]},
$$

where $\bar{\theta}=\frac{1}{2-2 \bar{\varepsilon}}$.

Then,

(a) $\left\{z_{k}\right\}$ converges weakly to a fixed point of $T$;

(b) $\left\{x_{k}\right\}$ converges weakly to $J_{\gamma B} z^{*} \in \operatorname{Zer}[A+B+C+(2-\alpha) I]$;

(c) $\left\{y_{k}\right\}$ converges weakly to $J_{\gamma B} z^{*} \in \operatorname{Zer}[A+B+C+(2-\alpha) I]$.

Proof. Observe that

$$
z_{k+1}=\left(1-\lambda_{k}\right) w_{k}+\lambda_{k} T w_{k} .
$$

It follows from Lemma 3.4 that $T$ is $\bar{\theta}$-averaged. Then, there exists a nonexpansive operator $T_{1}$ such that $T=(1-\bar{\theta}) I+\bar{\theta} T_{1}$. It follows that

$$
\begin{aligned}
z_{k+1} & =\left(1-\lambda_{k}\right) w_{k}+\lambda_{k}\left((1-\bar{\theta}) w_{k}+\bar{\theta} T_{1} w_{k}\right) \\
& =\left(1-\lambda_{k} \bar{\theta}\right) w_{k}+\lambda_{k} \bar{\theta} T_{1} w_{k} .
\end{aligned}
$$

Using Lemma 2.1, we obtain that, for any $z \in F i x\left(T_{1}\right), \lim _{k \rightarrow \infty}\left\|z_{k}-z\right\|$ exists. Moreover, $\sum_{k=0}^{\infty}\left\|z_{k+1}-z_{k}\right\|^{2}<+\infty$ and $\left\{z_{k}\right\}$ converges weakly to a point in $\operatorname{Fix}\left(T_{1}\right)$.

(a) Since $F i x(T)=F i x\left(T_{1}\right)$, we get $\left\{z_{k}\right\}$ converges weakly to a fixed point of $T$.

(b) Letting $z^{*} \in \operatorname{Fix}(T)$, we have

$$
\left\|z_{k+1}-z^{*}\right\|^{2}=\left(1-\lambda_{k}\right)\left\|w_{k}-z^{*}\right\|^{2}+\lambda_{k}\left\|T w_{k}-z^{*}\right\|^{2}-\lambda_{k}\left(1-\lambda_{k}\right)\left\|T w_{k}-w_{k}\right\|^{2} .
$$

In addition, from (3.5), we have

$$
\begin{aligned}
\left\|T w_{k}-z^{*}\right\|^{2} \leq & \left\|w_{k}-z^{*}\right\|^{2}-\frac{1-\bar{\theta}}{\bar{\theta}}\left\|T w_{k}-w_{k}\right\|^{2} \\
& -\gamma\left(2 \beta-\frac{\gamma}{\bar{\varepsilon}}\right)\left\|C \circ J_{\gamma B} w_{k}-C \circ J_{\gamma B} z^{*}\right\|^{2} \\
& -\gamma(2-\alpha)\left[2-\frac{\gamma(2-\alpha)}{\bar{\varepsilon}}\right]\left\|J_{\gamma B} w_{k}-J_{\gamma B} z^{*}\right\|^{2} .
\end{aligned}
$$

From (4.3) and (4.4), we get

$$
\begin{aligned}
\left\|w_{k}-z^{*}\right\|^{2} \geq & \left\|z_{k+1}-z^{*}\right\|^{2}+\left(1-\bar{\theta} \lambda_{k}\right) \frac{\lambda_{k}}{\bar{\theta}}\left\|T w_{k}-w_{k}\right\|^{2} \\
& +\gamma \lambda_{k}\left(2 \beta-\frac{\gamma}{\varepsilon}\right)\left\|C J_{\gamma B} w_{k}-C J_{\gamma B} z^{*}\right\|^{2} .
\end{aligned}
$$

Now, we prove $\lim _{k \rightarrow+\infty}\left\|w_{k}-z^{*}\right\|=\lim _{k \rightarrow+\infty}\left\|z_{k}-z^{*}\right\|$. Observe that

$$
\begin{aligned}
\left\|w_{k}-z^{*}\right\| & =\left\|z_{k}+\delta_{k}\left(z_{k}-z_{k-1}\right)-z^{*}\right\| \\
& \leq\left\|z_{k}-z^{*}\right\|+\delta_{k}\left\|z_{k}-z_{k-1}\right\|
\end{aligned}
$$

and

$$
\begin{aligned}
\left\|z_{k+1}-z^{*}\right\| & =\left\|\left(1-\lambda_{k}\right)\left(w_{k}-z^{*}\right)+\lambda_{k}\left(T w_{k}-z^{*}\right)\right\| \\
& \leq\left\|w_{k}-z^{*}\right\| .
\end{aligned}
$$

Since $\lim _{k \rightarrow+\infty}\left\|z_{k}-z_{k-1}\right\|=0$ and $\lim _{k \rightarrow+\infty}\left\|z_{k}-z^{*}\right\|$ exists, we conclude from (4.6) and (4.7) that $\lim _{k \rightarrow+\infty}\left\|w_{k}-z^{*}\right\|=\lim _{k \rightarrow+\infty}\left\|z_{k}-z^{*}\right\|$. From (4.5), we obtain $\lim _{k \rightarrow+\infty}\left\|T w_{k}-w_{k}\right\|=0$, 
and $\lim _{k \rightarrow+\infty}\left\|C J_{\gamma B} w_{k}-C J_{\gamma B} z^{*}\right\|=0$. Now, we set $x_{k}=J_{\gamma B}\left(w_{k}\right), y_{k}=J_{\gamma A}\left\{[2-\gamma(2-\alpha)] x_{k}-\right.$ $\left.w_{k}-\gamma C x_{k}\right\}, u_{k}=\frac{1}{\gamma}\left(w_{k}-x_{k}\right) \in B x_{k}$ and $v_{k}=\frac{1}{\gamma}\left\{[2-\gamma(2-\alpha)] x_{k}-w_{k}-\gamma C x_{k}-y_{k}\right\} \in A y_{k}$. Notice that

$$
\begin{aligned}
\left\|x_{k}-J_{\gamma B}\left(z^{*}\right)\right\| & =\left\|J_{\gamma B}\left(w_{k}\right)-J_{\gamma B}\left(z^{*}\right)\right\| \\
& \leq\left\|w_{k}-z^{*}\right\| \\
& \leq\left\|z_{k}-z^{*}\right\|+\delta_{k}\left\|z_{k}-z_{k-1}\right\| \\
& \leq\left\|z_{k}-z^{*}\right\|+\delta\left\|z_{k}-z_{k-1}\right\|,
\end{aligned}
$$

$\lim _{k \rightarrow+\infty}\left\|z_{k}-z_{k-1}\right\|=0$ and $\lim _{k \rightarrow+\infty}\left\|z_{k}-z^{*}\right\|$ exists. Then, we obtain that the sequence $\left\{x_{k}\right\}_{k \geq 0}$ is bounded and there exists a subsequence $\left\{x_{k_{j}}\right\}$ and a weak sequential cluster point $\bar{w}$ such that $x_{k_{j}} \rightarrow \bar{w}$. Letting $x^{*}=J_{\gamma B}\left(z^{*}\right)$, we have $x^{*} \in \operatorname{Zer}[A+B+C+(2-\alpha) I]$. Since $C$ is maximally monotone, $C x_{k} \rightarrow C x^{*}$ and $x_{k_{j}} \rightarrow \bar{w}$, we conclude from the weak-to-strong sequential closeness of $C$ that $C \bar{w}=C x^{*}$ and thus $C x_{k_{j}} \rightarrow C \bar{w}$. In view of $y_{k}-x_{k}=T w_{k}-w_{k} \rightarrow 0$ as $k \rightarrow \infty$, we see that

$$
\begin{aligned}
& x_{k_{j}} \rightarrow \bar{w}, \quad y_{k_{j}} \rightarrow \bar{w}, \quad u_{k_{j}} \rightarrow \frac{1}{\gamma}\left(z^{*}-\bar{w}\right), \\
& v_{k_{j}} \rightarrow \frac{1}{\gamma}\left\{[1-\gamma(2-\alpha)] \bar{w}-z^{*}-\gamma C \bar{w}\right\} \quad \text { as } j \rightarrow \infty .
\end{aligned}
$$

Since $\left(y_{k_{j}}, v_{k_{j}}\right) \in \operatorname{Graph}(A),\left(x_{k_{j}}, u_{k_{j}}\right) \in \operatorname{Graph}(B),\left(x_{k_{j}}, C x_{k_{j}}\right) \in \operatorname{Graph}(C)$ and $\left(x_{k_{j}},(2-\alpha) x_{k_{j}}\right) \in$ $\operatorname{Graph}[(2-\alpha) I]$, Lemma 2.4 guarantees that $\bar{w} \in \operatorname{Zer}[A+B+C+(2-\alpha) I]$. Hence, $\bar{w}=J_{\gamma B}\left(z^{*}\right)$. Therefore $\bar{w}$ is the unique weak sequential point of $\left\{x_{k}\right\}_{k \geq 0}$, and $\left\{x_{k}\right\}_{k \geq 0}$ converges weakly to $J_{\gamma B}\left(z^{*}\right)$.

(c) Since $y_{k}-x_{k}=T w_{k}-w_{k} \rightarrow 0$ as $k \rightarrow \infty$ and $x_{k} \rightarrow J_{\gamma B}\left(z^{*}\right)$, we obtain $y_{k} \rightarrow J_{\gamma B}\left(z^{*}\right)$ immediately.

\subsection{The regularization of the inertial parameterized three-operator algorithm.}

Algorithm 4.2. Initialization: Set $z_{0} \in H, 0<\gamma<\min \left\{\frac{1}{2-\alpha}, \beta\right\}$, and sequence $\left\{\lambda_{k}\right\}_{k \geq 0} \in$ $\left(0,2-2 \max \left\{\frac{\gamma}{2 \beta}, \frac{\gamma(2-\alpha)}{2}\right\}\right)$. Choose $\left\{\delta_{k}\right\} \in[0,1)$.

Iterative steps: For $k=0,1, \cdots$, iterate:

Step 1. Compute

$$
w_{k}=z_{k}+\delta_{k}\left(z_{k}-z_{k-1}\right) \text {. }
$$

Step 2. Compute

$$
x_{k}=J_{\gamma B}\left(w_{k}\right) .
$$

Step 3. Compute

$$
y_{k}=J_{\gamma A}\left\{\left[2-\gamma\left(2-\alpha_{n}\right)\right] x_{k}-w_{k}-\gamma C x_{k}\right\} .
$$

Step 4. Compute

$$
z_{k+1}=w_{k}+\lambda_{k}\left(x_{k}-y_{k}\right) .
$$

Theorem 4.2. Let $A, B$ be maximally monotone operators from $H$ to $2^{H}$ with $0 \in$ ri(domAdomB) and let $C$ be a cocoercive operator. Let $\left\{\alpha_{n}\right\}_{n \in N}$ be an increasing sequence in $(1,2)$ such that $\lim _{n \rightarrow+\infty} \alpha_{n}=2$. Then the sequence $\left\{z_{k}\right\}_{k \in N}$ generated by Algorithm 4.2 converges to $z_{n}^{*}$ such that $J_{\gamma B} z_{n}^{*} \in \operatorname{Zer}\left[A+B+C+\left(2-\alpha_{n}\right) I\right]$, and

(1) $\lim _{n \rightarrow+\infty} J_{\gamma B} z_{n}^{*}=P_{Z e r(A+B+C)}(0)$; 
(2) suppose $\left\{z_{n}^{*}\right\}_{n \in N}$ is a convergent sequence, say, $\lim _{n \rightarrow+\infty} z_{n}^{*}=z^{*}$. Then, $J_{\gamma B} z^{*}$ is a solution to $0 \in A x+B x+C x$, and $\left\|J_{\gamma B} z^{*}\right\| \leq\|y\|$ for any $y \in \operatorname{Zer}(A+B+C)$.

This proof is analogous to Theorem 3.2. So, we omit the proof here.

\section{NUMERICAL RESULTS}

In this section, we present an example to demonstrate the feasibility of the parameterized three-operator algorithm and the inertial parameterized three-operator algorithm.

All experiments are written in Matlab and performed on a personal computer Intel(R) core(TM) i5-10210U CPU @ 1.60GHz 2.11GHz, RAM 16.00GB. 'CPU time' and 'Iter.' denote the cpu time in seconds and the number of iterations.

Example 5.1. Let $C_{1}$ be a circle at $(5,0)$ with radius $2, C_{2}$ be a box centred at $(5,0)$ with side length 2 , and

$$
A_{1}=\left(\begin{array}{cc}
0 & 1 \\
0 & 1
\end{array}\right), b=\left(\begin{array}{l}
0 \\
1
\end{array}\right) .
$$

A simple minimization problem is to solve

$$
\min _{x \in C_{1} \cap C_{2}} \frac{1}{2}\left\|A_{1} x-b\right\|^{2}=\min _{x \in \mathbb{R}^{2}} \frac{1}{2}\left\|A_{1} x-b\right\|^{2}+l_{C_{1}}(x)+l_{C_{2}}(x),
$$

where the indicator functions $l_{C_{1}}$ and $l_{C_{2}}$ are

$$
\iota_{C_{1}}(x)=\left\{\begin{array}{lr}
0, & x \in C_{1}, \\
+\infty, & \text { otherwise, }
\end{array} \quad \text { and } \quad l_{C_{2}}(x)= \begin{cases}0, & x \in C_{2}, \\
+\infty, & \text { otherwise } .\end{cases}\right.
$$

Problem (5.2) is equivalent to the problem of finding $x \in \mathbb{R}^{2}$ such that

$$
0 \in N_{C_{1}}(x)+N_{C_{2}}(x)+A_{1}^{*}\left(A_{1} x-b\right),
$$

that is, $A=N_{C_{1}}(x), B=N_{C_{2}}(x)$, and $C=A_{1}^{*}\left(A_{1} x-b\right)$ in problem (1.1).

First, let us make the initial values randomly and take $\left\|x_{k+1}-x_{k}\right\|<\varepsilon=10^{-7}$ as the stopping criteria. Let $\alpha_{n}$ equal to 2 and $2-\frac{1}{50}$. If the initial values vary, we get the different $x_{n}^{*}$ by the classic three-operator algorithm and the same $x_{n}^{*}$ by the parameterized three-operator algorithm in Table 1.

TABLE 1 . The result with different $z_{0}$

\begin{tabular}{l|c|cc}
\hline$z_{0}$ & $\alpha_{n}$ & $x_{n}^{*}$ & $\left\|x_{n}^{*}\right\|$ \\
\hline$(-16.31170420,99.54719595)$ & 2 & $(4.88170026,0.50000016)$ & 4.90723930 \\
& $2-\frac{1}{50}$ & $(3.06350833,0.50000002)$ & 3.10404306 \\
\hline$(60.05609378,-71.62273227)$ & 2 & $(5.00000000,0.49999992)$ & 5.02493780 \\
& $2-\frac{1}{50}$ & $(3.06350835,0.50000008)$ & 3.10404309 \\
\hline$(-31.14813983,-2.85766428)$ & 2 & $(3.45500796,0.50000014)$ & 3.49099987 \\
& $2-\frac{1}{50}$ & $(3.06350834,0.50000006)$ & 3.10404308 \\
\hline$(-44.61540301,-90.76572187)$ & 2 & $(3.29256549,0.50000011)$ & 3.33031344 \\
& $2-\frac{1}{50}$ & $(3.06350834,0.50000004)$ & 3.10404307 \\
\hline$(53.10335763,59.03998023)$ & & $(5.00000000,0.50000011)$ & 5.02493782 \\
& $2-\frac{1}{50}$ & $(3.06350833,0.50000003)$ & 3.10404306 \\
\hline \hline
\end{tabular}


TABLE 2. The result of the parameterized three-operator algorithm

\begin{tabular}{l|cccc}
\hline$\alpha_{n}$ & $x_{n}^{*}$ & $\left\|x_{n}^{*}\right\|$ & CPU time & Iter. \\
\hline 1 & $(3.0635083410,0.5000000547)$ & 3.10404307 & 0.0128618 & 93 \\
$2-\frac{1}{2}$ & $(3.0635083290,0.5000000080)$ & 3.10404306 & 0.0113363 & 89 \\
$2-\frac{1}{3}$ & $(3.0635083080,0.4999999269)$ & 3.10404302 & 0.0145410 & 89 \\
$2-\frac{1}{4}$ & $(3.0635083210,0.4999999771)$ & 3.10404304 & 0.0135360 & 91 \\
$2-\frac{1}{5}$ & $(3.0635083142,0.4999999509)$ & 3.10404303 & 0.0125821 & 92 \\
$2-\frac{1}{6}$ & $(3.0635083255,0.4999999945)$ & 3.10404305 & 0.0103691 & 94 \\
$2-\frac{1}{7}$ & $(3.0635083216,0.4999999794)$ & 3.10404304 & 0.0120192 & 95 \\
$2-\frac{1}{8}$ & $(3.0635083272,0.5000000010)$ & 3.10404305 & 0.0146272 & 97 \\
$2-\frac{1}{9}$ & $(3.0635083256,0.4999999951)$ & 3.10404305 & 0.0125123 & 98 \\
$2-\frac{1}{10}$ & $(3.0635083282,0.5000000051)$ & 3.10404305 & 0.0118059 & 100 \\
$2-\frac{1}{50}$ & $(3.0635083259,0.4999999961)$ & 3.10404305 & 0.0134067 & 161 \\
$2-\frac{1}{100}$ & $(3.0635083141,0.4999999504)$ & 3.10404303 & 0.0115740 & 238 \\
$2-\frac{1}{1000}$ & $(3.0635083182,0.4999999662)$ & 3.10404304 & 0.0133408 & 1634 \\
$2-\frac{1}{10000}$ & $(3.0635083315,0.5000000177)$ & 3.10404306 & 0.1660751 & 15611 \\
\hline \hline
\end{tabular}

TABLE 3. The result of the inertial parameterized three-operator algorithm

\begin{tabular}{l|cccc}
\hline$\alpha_{n}$ & $x_{n}^{*}$ & $\left\|x_{n}^{*}\right\|$ & CPU time & Iter. \\
\hline 1 & $(3.0635141757,0.5000226518)$ & 3.10405247 & 0.0063132 & 42 \\
$2-\frac{1}{2}$ & $(3.0635083002,0.4999998968)$ & 3.10404301 & 0.0033579 & 48 \\
$2-\frac{1}{3}$ & $(3.0635083563,0.5000001139)$ & 3.10404310 & 0.0037321 & 49 \\
$2-\frac{1}{4}$ & $(3.0635082886,0.4999998516)$ & 3.10404299 & 0.0065120 & 49 \\
$2-\frac{1}{5}$ & $(3.0635083600,0.5000001281)$ & 3.10404311 & 0.0072611 & 50 \\
$2-\frac{1}{6}$ & $(3.0635083297,0.5000000109)$ & 3.10404306 & 0.0068858 & 51 \\
$2-\frac{1}{7}$ & $(3.0635083137,0.4999999489)$ & 3.10404303 & 0.0068482 & 51 \\
$2-\frac{1}{8}$ & $(3.0635082929,0.4999998682)$ & 3.10404300 & 0.0093895 & 52 \\
$2-\frac{1}{9}$ & $(3.0635082796,0.4999998169)$ & 3.10404298 & 0.0057680 & 52 \\
$2-\frac{1}{10}$ & $(3.0635083406,0.5000000529)$ & 3.10404307 & 0.0081525 & 53 \\
$2-\frac{1}{50}$ & $(3.0635083658,0.5000001506)$ & 3.10404311 & 0.0067789 & 57 \\
$2-\frac{1}{100}$ & $(3.0635082950,0.4999998763)$ & 3.10404300 & 0.0068986 & 58 \\
$2-\frac{1}{1000}$ & $(3.0635083640,0.5000001438)$ & 3.10404311 & 0.0069941 & 70 \\
$2-\frac{1}{10000}$ & $(3.0635083418,0.5000000577)$ & 3.10404308 & 0.0076828 & 104 \\
\hline \hline
\end{tabular}

To generate an initial randomly, $z_{0}=[-16.3117042099 .54719595]$, for the parameterized three-operator algorithm, the optimal solution $x_{n}^{*}$ does not varies when we use different values of $\alpha_{n}$ in Table 2. For the inertial parameterized three-operator algorithm, the optimal solution $x_{n}^{*}$ does not varies when we use different values of $\alpha_{n}$ in Table 3. Comparing the inertial parameterized three-operator algorithms with the parameterized three-operator algorithm, we can see that the CPU time and the steps number of iterations have been greatly improved. The parameterized three-operator algorithm and the inertial parameterized three-operator algorithm always yield the unique least norm solution. In addition, the algorithm with the inertia is more advantageous and feasible than the one without the inertia. 


\section{Acknowledgments}

This paper was supported by the Fundamental Research Funds for the Central Universities (No. 3122018L004).

\section{REFERENCES}

[1] T.H. Cuong, J.C. Yao, N.D. Yen, Qualitative properties of the minimum sum-of-squares clustering problem, Optimization, 69 (2020), 2131-2154.

[2] P. Cubiotti, J.C. Yao, On the Cauchy problem for a class of differential inclusions with applications, Appl. Anal. 99 (2020), 2543-2554.

[3] D. Davis, W. Yin, A Three-Operator Splitting Scheme and Its Optimization Applications, Tech. Rep CAM 15-13. University of California, Los Angeles, 2015.

[4] X. Qin, N.T. An, Smoothing algorithms for computing the projection onto a Minkowski sum of convex sets, Comput. Optim. Appl. 74 (2019), 821-850.

[5] D.R. Sahu, J.C. Yao, M. Verma, K.K. Shukla, Convergence rate analysis of proximal gradient methods with applications to composite minimization problems, Optimization, 70 (2021), 75-100.

[6] J. Douglas, H.H. Rachford, On the numberical solution of heat conduction problems in two and three space variables, Trans. Amer. Math. Soc. 82 (1956), 421-439.

[7] X. Qin, S.Y. Cho, L. Wang, A regularization method for treating zero points of the sum of two monotone operators, Fixed Point Theory Appl. 2014 (2014), Article ID 75.

[8] S.S. Chang, C.F. Wen, J.C. Yao, Zero point problem of accretive operators in Banach spaces, Bull. Malaysian Math. Sci. Soc. 42 (2019), 105-118.

[9] X. Qin, S.Y. Cho, L. Wang, Strong convergence of an iterative algorithm involving nonlinear mappings of nonexpansive and accretive type, Optimization, 67 (2018), 1377-1388.

[10] E.K. Ryu, Y. Liu, W. Yin, Douglas-Rachford splitting and ADMM for pathological convex optimization, Comput. Optim. Appl. 74 (2019), 747-778.

[11] H. Raguet, A note on the forward-Douglas-Rachford splitting for monotone inclusion and convex optimization, Optim. Lett. 13 (2019), 717-740.

[12] B.F. Svaiter, On weak convergence of the Douglas-Rachford method, SIAM J. Control. Optim. 49 (2011), 280-287.

[13] S.Y. Cho, X. Qin, L. Wang, Strong convergence of a splitting algorithm for treating monotone operators, Fixed Point Theory Appl. 2014 (2014), Article ID 94.

[14] D. Wang, X. Wang, A parameterized Douglas-Rachford algorithm, Comput. Optim. Appl. 164 (2019), 263-284.

[15] L.M. Briceño-Arias, Forward-Douglas-Rachford splitting and forward-partial inverse method for solving monotone inclusions, Optim. 60 (2015), 1239-1261.

[16] F.Y. Cui, Y.C. Tang, Y. Yang, An inertial three-operator splitting algorithm with applications to image inpainting, Appl. Set-Valued. Anal. Optim. 164 (2017), 263-284.

[17] D. Davis, W.T. Yin, A three-operator splitting scheme and its optimization applications, Set-Valued. Var. Anal. 25 (2017), 829-858.

[18] D.V. Hieu, L.V. Vy, P.K. Quy, Three-operator splitting algorithm for a class of variational inclusion problems, Bull. Iranian Math. Soc. 91 (2020), 1055-1071.

[19] A. Moudafi, A three-operator splitting algorithm for null-point problems, Fixed Point Theory 21 (2020), 685-692.

[20] C.X. Zong, Y.C. Tang, Y.J. Cho, Convergence analysis of an inexact three-operator splitting algorithm, Symmetry 10 (2018), 563.

[21] B.T. Polyak, Some methods of speeding up the convergence of iteration methods, U.S.S.R. Comput. Math. Math. Phys. 4 (1964), 1-17.

[22] X. Qin, L. Wang, J.C. Yao, Inertial splitting method for maximal monotone mappings, J. Nonlinear Convex Anal. 21 (2020), 2325-2333.

[23] Y. Shehu, P. Cholamjiak, Iterative method with inertial for variational inequalities in Hilbert spaces, Calcolo, 56 (2019), 4. 
[24] Y. Shehu, J.C. Yao, Rate of convergence for inertial iterative method for countable family of certain quasinonexpansive mappings, J. Nonlinear Convex Anal. 21 (2020), 533-541.

[25] S.Y. Cho, A monotone Bregan projection algorithm for fixed point and equilibrium problems in a reflexive Banach space, Filomat, 34 (2020), 1487-1497.

[26] J. Zhao, C. Zhang, Improved relaxed CQ method with inertial acclerated technique for the split feasibility problem, J. Nonlinear Convex Anal. 21 (2020), 2391-2402.

[27] R.I. Bot, E.R. Csetnek, C. Hendrich, Inertial Douglas-Rachford splitting for monotone inclusion problems, Appl. Math. Comput. 256 (2015), 472-487.

[28] H.H. Bauschke, P.L. Combettes, Convex Analysis and Monotone Operator Theory in Hilbert Spaces, Second Edition, Springer, 2017.

[29] H. Brézis, Chapitre П Operateurs Maximaux Monotones, North-Holland Math Stud. 5 (1973), 19-51. 